\title{
Breathing-controlled electrical stimulation could modify the affective component of neuropathic pain after amputation: a case report
}

This article was published in the following Dove Press journal:

Journal of Pain Research

II April 2012

Number of times this article has been viewed

\author{
Sheng $\mathrm{Li}^{1,2}$ \\ Danielle H Melton' \\ Jeffrey C Berliner' \\ 'Department of Physical Medicine \\ and Rehabilitation, University of \\ Texas Medical School - Houston, \\ Houston, TX; ${ }^{2}$ UTHealth Motor \\ Recovery Laboratory, Institute \\ for Rehabilitation and Research, \\ Memorial Hermann Hospital, \\ Houston, TX, USA
}

\begin{abstract}
In this case, a 31-year-old male suffered phantom neuropathic pain for more than 3 years after an above-the-knee amputation. His shooting phantom pain disappeared after the first session of breathing-controlled electrical stimulation, and reappeared or was triggered 28 days after an experimental error during which he received sustained electrical stimulation. In other words, painful shooting stimuli may not have been "cured" but forgotten and retriggered by a fearful event due to the experimental error. Therefore, this accidental finding provides a unique opportunity to understand sensory and affective components of neuropathic pain, and a novel intervention could modify the affective component of it.
\end{abstract}

Keywords: neuropathic pain, amputation, electrical stimulation, voluntary breathing

\section{Introduction}

Neuropathic pain, including phantom pain, is a chronic condition caused by a primary lesion or dysfunction of the nervous system. ${ }^{1}$ Neuropathic pain is very common. ${ }^{2}$ About $60 \%$ of patients with limb amputation have phantom pain at 2 years postamputation. The pain does not change after 6 months postamputation. ${ }^{3}$ Neuropathic pain is multidimensional, including sensory and affective components. ${ }^{4}$ When superficial tactile stimulation is applied to the hand area where acupuncture points are located, activation is seen only in somatosensory cortices. However, when the acupuncture points are stimulated and pain is experienced by the subjects, activation of additional cortical areas, such as anterior cingulate cortex and insula, is observed. ${ }^{5}$ Sensation (painful stimuli) and perception of pain are possibly two different but parallel processes. ${ }^{4}$

Neuropathic pain is difficult to manage and is associated with poorer physical, psychological, and social functioning. ${ }^{6-8}$ Many nonpharmacological modalities have been used for management of neuropathic pain with different mechanisms. Transcutaneous electrical nerve stimulation has been used for pain relief. ${ }^{9}$ The possible mechanisms are the gate control theory ${ }^{10}$ and the release of endogenous opioids. ${ }^{11,12}$ The well-accepted mechanism for acupuncture-related pain relief is the release of endogenous opioids, which is reversed by naloxone (opioid-antagonist). ${ }^{13,14}$ Recently, it has been found that repetitive painful stimulation (aversiveness) leads to significant pain attenuation. The induced pain attenuation is not naloxone-reversible. ${ }^{15}$ Given our recent discovery of systemic effects of voluntary breathing, including reduction in muscle tone, ${ }^{16,17}$ here we report an innovative intervention that integrates all these modalities by voluntary breathing, ie, breathing-controlled aversive electrical stimulation (BreEStim), to acupuncture points for neuropathic pain management. We hypothesized 
that BreEStim would have a greater analgesic effect than electrical stimulation alone (EStim), because it integrates the aforementioned different pain-modifying mechanisms.

\section{Case description}

The subject was a 31-year-old white male. He had a work-related traumatic injury to his left leg 3.5 years ago that resulted in an above-the-knee amputation. At the time of study he was working full time. He had no skin issues with the residual limb and usually wore his prosthesis about 12-14 hours a day with a 1-hour break. He could walk several blocks without a break. However, he had severe and constant pain and he had to schedule his work break around his pain medication schedules. Pain was usually constant 6-7/10, which decreased to 3-4/10 when medicated. Characteristics of phantom pain were mainly burning and electrical shooting down to the phantom foot. His pain has been stable on the pain regime for 4 weeks. He was asked not to change his pain regime during the experiment. The patient had no other medical issues.

The subject provided informed consent prior to participation in the study, according to procedures approved by our institutional review board. The subject was planned to undertake the following experimental protocol: treatment (five consecutive days) - washout (1 week) - control (five consecutive days) - washout (1 week) - treatment (5 days) - followup (1 week). Treatment was BreEStim at the self-selected aversive (painful) intensity. Each BreEStim session consisted of 120 stimuli, which usually took about 30-40 minutes including breaks between stimulation trials. In control, the subject received only EStim during normal breathing. This within-subject crossover design allowed comparisons of effectiveness in reduction of neuropathic pain between BreEStim and EStim. The washout/follow-up period allowed washout of previous intervention, as well as assessment of possible accumulative or carry-over effect.

The experimental set-up was adopted from our recently published protocol. ${ }^{17}$ The subject was seated comfortably. The arms and hands were placed comfortably on the experimental table in approximately symmetrical positions. Surface electrodes were placed over acupuncture points Neiguan and Weiguan on the ipsilateral forearm. ${ }^{18}$ The intensity of electrical stimulation was started from 0 , gradually increased to the highest level as tolerated using an electrical stimulator (Digitimer DS7A; Welwyn Garden City, England). Aversiveness of electrical stimulation is part of treatment strategy. However, the intensity of electrical stimulation was self-selected.
During EStim, a single-pulse electrical stimulus (width $0.1 \mathrm{~ms}$, square wave) was delivered to the forearm through surface electrodes randomly every 4-7 seconds. During BreEStim, the subject was asked to wear a face mask. The face mask was connected indirectly to the experimental computer via a pneumotach system (Hans Rodolph, Inc). A stimulus was triggered if the airflow reached or exceeded $40 \%$ of maximum airflow during a volitionally effortful inhalation. ${ }^{17}$ Rest was taken upon request. During the entire experimental period (4 weeks), the subject maintained the same dose and schedule of pain medications. Both BreEStim and EStim treatment sessions were performed at the same time of the day (between 11 am and noon), such that changes in the pain rating could possibly be attributed to stimulation effects and not diurnal variation.

Voluntary inhalation played an important role in this intervention. Voluntary inhalation was defined as effortful deep and fast inhalation. The subject was instructed to take a single isolated deep breath, similar to routine deep breaths, but faster and stronger, usually involving obvious expansion of the chest wall. It has been reported that there are respiratory-specific connections between the insula and the activity of pulmonary stretch receptors. ${ }^{19}$ When wearing a face mask, the subject tolerated such breathing very well. No hyperventilation was reported. ${ }^{17}$

The primary outcome measurement included visual analog scores (VAS) and modified visual analog scores (mVAS). VAS has been extensively used and validated. ${ }^{20}$ mVAS further quantifies the effect of pain reduction by measuring duration and amount of change in VAS, ie, how much pain was reduced and how long it lasted (reduction $\times$ hours). The intensity of electrical stimulation was recorded daily during the course of treatment. The averaged intensity for each session was used. The secondary outcome measurement was General Well-Being Schedule Survey and Center for Epidemiologic Studies Depression Scale National Institute of Mental Health Survey to examine the possible effect of pain reduction on general well-being, depression, and quality of life of the patients. These surveys have been well validated and commonly used. ${ }^{21-23}$ Any side effects, tolerance of voluntary breathing via a face mask, and psychosocial effects were recorded.

The intensity of electrical stimulation (the lower panels) was comparable between BreEStim and EStim interventions (Figure 1). Overall, BreEStim had greater analgesic effects than EStim (the upper panels). On average, BreEStim produced a 2-4 point reduction on VAS for 3-4 hours after each session. EStim produced a 0-2 point reduction on 


\section{BreEStim}

Effect (reduction $\times$ hours)

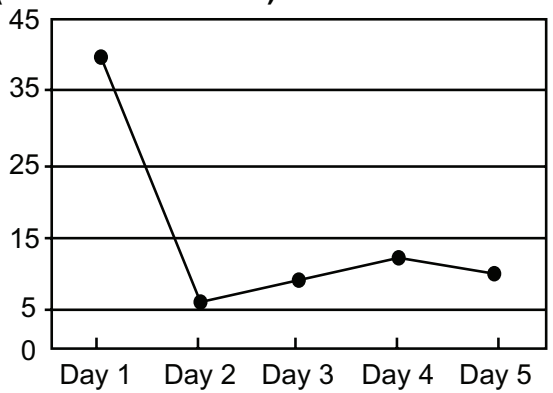

Stimulation intensity ( $\mathrm{mA}$ )

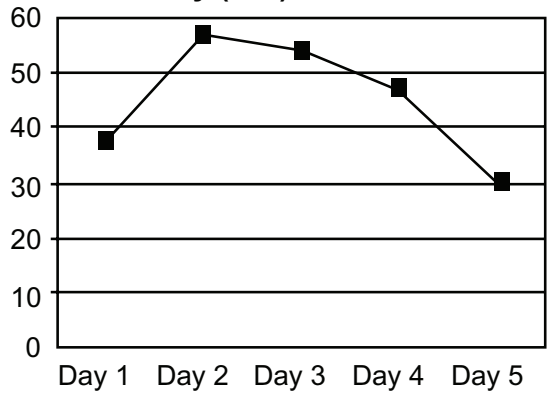

\section{EStim}

Effect (reduction $\times$ hours)

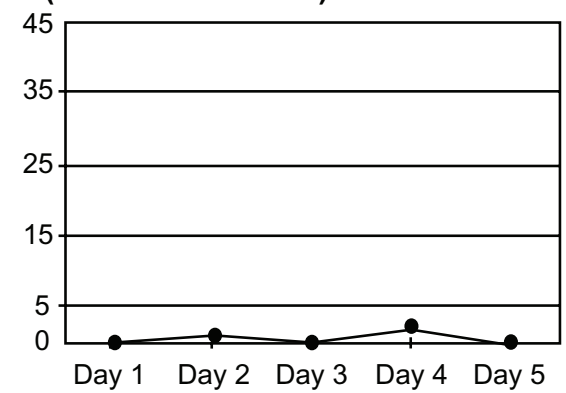

Stimulation intensity (mA)

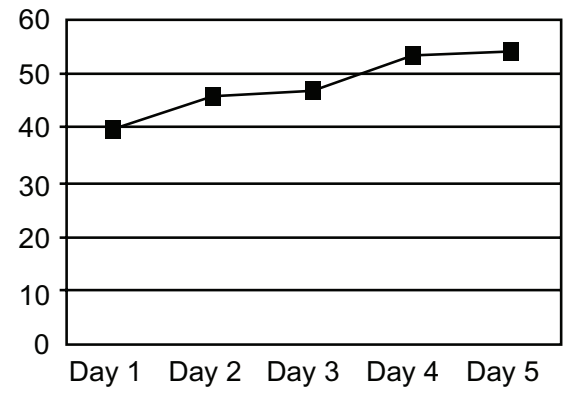

Figure I Comparison of analgesic effect between breathing-controlled aversive electrical stimulation (BreEStim) and electrical stimulation only (EStim).

VAS for 1-2 hours. The patient reported that his baseline pain level was $5-7 / 10$ prior to each treatment session.

The subject was excited that his intense and constant shooting phantom pain disappeared after BreEStim on Day 1, despite the fact that the sensation of phantom leg/ foot remained. The shooting phantom pain disappeared after the BreEStim protocol (1 week) or at follow-up after EStim (a total of 4 weeks). His baseline pain remained the same, mainly burning and aching in nature. The subject tolerated the first BreEStim and EStim sessions well. Unfortunately, the subject did not receive the second BreEStim session, because of the experimental error. During reassembling of the experimental setting prior to the second BreEStim session, one channel was left unwired. The subject received sustained electrical stimulation for approximately 5 seconds (supposed to be $0.1 \mathrm{~ms}$ ) at the aversive level during the first BreEStim trial on Day 1 of the planned second 5-day BreEStim treatment. He felt fearful and anxious about further BreEStim trials and reported that his shooting pain came back as constant 7/10. The study discontinued at that point. One of the authors also tried and experienced the same sustained electrical stimulation during normal breathing, and felt transient pain on the stimulated area for about an hour. During a follow-up clinic visit 4 weeks later, the subject reported that the baseline burning pain remained the same, while the shooting pain gradually subsided to $2 / 10$, lasting about
1 minute every 3-4 days. He also reported no change in his pain regime. The subject showed no carryover effects after the intervention. No noticeable overall change in quality of life was found based on General Well-Being and Center for Epidemiologic Studies Depression Scale surveys, although the subject reported that he felt great during and after each BreEStim along with pain reduction.

\section{Discussion}

This case demonstrates that BreEStim has greater and longer analgesic effects than EStim on chronic phantom pain. This is possibly attributed to the fact that BreEStim has integrated different pain-modifying mechanisms into one intervention, including the following:

- Electroacupuncture effect. Acupuncture-related analgesic effects that are naloxone-reversible are triggered when acupuncture points are stimulated., ${ }^{54}$

- Habituation to aversive stimuli. Habituation to aversive stimuli occurs and is not naloxone-reversible. ${ }^{15}$ Therefore, the two mechanisms could have additive analgesic effects. The intensity was about the same between BreEStim and EStim groups (Figure 1). It is thus less likely that aversive electrical stimulation plays a primary and/or placebo role in pain reduction.

- Influence of voluntary breathing. Effortful inspiration and expiration requires extensive cortical and 
subcortical activation. The activation is more extensive during voluntary inspiration than voluntary expiration. The insular cortex is consistently activated during various sensorimotor respiratory tasks. ${ }^{25}$ The insular cortex is critical in affective processing of pain. ${ }^{26}$

- Anterograde amnesia to aversive stimulation. When aversive stimulation is delivered during activation of the insular cortex, item-specific anterograde amnesia to the stimulation occurs. ${ }^{27}$ In other words, unpleasantness of peripheral noxious stimulation is not remembered or decreased immediately after voluntary breathingcontrolled electrical stimulation, when the insular cortex is activated during voluntary breathing. As such, it is expected to facilitate habituation to aversive stimulation even at a higher intensity, subsequently increasing the analgesic effect of stimulation, thus a positive feedback loop. This is likely the case during BreEStim, because the subject requested a higher intensity of electrical stimulation gradually during each session, even noxious, for a better analgesic effect.

- The reward system is triggered. As described, this positive feedback loop is likely to occur by triggering the reward system. ${ }^{28}$ As observed, the intensity of aversive electrical stimulation is driven higher gradually during the course of stimulation. However, a large-scale clinical study, possibly with imaging studies, such as functional magnetic resonance imaging, is needed to corroborate the findings and underlying mechanisms.

The accidental finding of disappearance and reappearance of shooting phantom pain associated with BreEStim provides a unique opportunity to advance our understanding of the mechanisms underlying postamputation neuropathic pain. This finding implies that shooting stimulus does not change, but it is not perceived as aversive after the first BreEStim. However, aversive perception of shooting stimulus is likely retriggered by an unexpected, fearful sustained electrical stimulation in the second BreEStim. It has been reported earlier that when associated with a negative emotional context, pain (eg, phantom pain after amputation) could be perceived as aversive and retriggered by a stressful life event. ${ }^{3}$ This accidental finding supports the idea that sensory and affective (unpleasant) components of neuropathic pain (phantom pain) are processed in parallel. ${ }^{4}$ It also suggests that BreEStim could modify the affective component, resulting in additional analgesic effects. The aforementioned anterograde amnesia ${ }^{27}$ may play an important role.

In summary, BreEStim appeared to provide greater analgesic effects than EStim alone in this case. The accidental findings associated with BreEStim support the concept that neuropathic pain is multidimensional. BreEStim could modify the affective component of neuropathic pain.

\section{Acknowledgment}

This study was supported in part by National Institutes of Health grants (NIH/NINDS R01NS060774; NIH/NICHD/ NCMRR R24 HD050821-08 under subcontract with Rehabilitation Institute of Chicago).

\section{Disclosure}

The authors report no conflicts of interest in this work.

\section{References}

1. Treede RD, Jensen TS, Campbell JN, et al. Neuropathic pain: redefinition and a grading system for clinical and research purposes. Neurology. 2008;70:1630.

2. Werhagen L, Budh CN, Hultling C, et al. Neuropathic pain after traumatic spinal cord injury - relations to gender, spinal level, completeness, and age at the time of injury. Spinal Cord. 2004;42:665.

3. Jensen TS, Krebs B, Nielsen J, et al. Immediate and long-term phantom limb pain in amputees: incidence, clinical characteristics and relationship to pre-amputation limb pain. Pain. 1985;21:267.

4. Price DD. Psychological and neural mechanisms of the affective dimension of pain. Science. 2000;288:1769-1772.

5. Hui KKS, Liu J, Makris N, et al. Acupuncture modulates the limbic system and subcortical gray structures of the human brain: evidence from fMRI studies in normal subjects. Human Brain Mapping. 2000;9:13.

6. Norrbrink Budh C, Hultling C, Lundeberg T. Quality of sleep in individuals with spinal cord injury: a comparison between patients with and without pain. Spinal Cord. 2005;43:85-95.

7. Stormer S, Gerner HJ, Gruninger W, et al. Chronic pain/dysaesthesiae in spinal cord injury patients: results of a multicentre study. Spinal Cord. 1997;35:446-455.

8. Jensen MP, Chodroff MJ, Dworkin RH. The impact of neuropathic pain on health-related quality of life: review and implications. Neurology. 2007;68:1178-1182.

9. Norrbrink Budh C, Lundeberg T. Non-pharmacological pain-relieving therapies in individuals with spinal cord injury: a patient perspective. Complement Ther Med. 2004;12:189-197.

10. Melzack R, Wall PD. Pain mechanisms: a new theory. Science. 1965;150:971-979.

11. Kalra A, Urban MO, Sluka KA. Blockade of opioid receptors in rostral ventral medulla prevents antihyperalgesia produced by transcutaneous electrical nerve stimulation (TENS). J Pharmacol Exp Ther. 2001;298:257-263.

12. Sluka KA, Deacon M, Stibal A, et al. Spinal blockade of opioid receptors prevents the analgesia produced by TENS in arthritic rats. J Pharmacol Exp Ther. 1999;289:840-846.

13. Huang C, Wang Y, Han JS, et al. Characteristics of electroacupunctureinduced analgesia in mice: variation with strain, frequency, intensity and opioid involvement. Brain Res. 2002;945:20-25.

14. Han JS. Acupuncture and endorphins. Neuroscience Letters. 2004; 361:258.

15. Rennefeld C, Wiech K, Schoell ED, et al. Habituation to pain: further support for a central component. Pain. 2010;148:503.

16. Li S, Laskin JJ. Influences of ventilation on maximal isometric force of the finger flexors. Muscle Nerve. 2006;34:651-655.

17. Li S, Rymer WZ. Voluntary breathing influences corticospinal excitability of nonrespiratory finger muscles. J Neurophysiol. 2011;105: $512-521$. 
18. Deadman P, Al-khafaji M, Baker K. A Manual of Acupuncture, 2nd ed. Visa, CA: Eastland Press; 2007.

19. Aleksandrov VG, Mercuriev VA, Ivanova TG, et al. Cortical control of Hering-Breuer reflexes in anesthetized rats. Eur J Med Res. 2009;14 Suppl 4:1-5.

20. McCarthy M Jr, Chang CH, Pickard AS, et al. Visual analog scales for assessing surgical pain. J Am Coll Surg. 2005;201:245-252.

21. Kooijman CM, Dijkstra PU, Geertzen JHB, et al. Phantom pain and phantom sensations in upper limb amputees: an epidemiological study. Pain. 2000;87:33.

22. Sherman RA, Sherman CJ, Parker L. Chronic phantom and stump pain among American veterans: results of a survey. Pain. 1984;18:83-95.

23. Wartan SW, Hamann W, Wedley JR, et al. Phantom pain and sensation among British veteran amputees. Br J Anaesth. 1997;78:652.
24. Wu MT, Sheen JM, Chuang KH, et al. Neuronal specificity of acupuncture response: a fMRI study with electroacupuncture. NeuroImage. 2002;16:1028.

25. Tsumori T, Yokota S, Kishi T, et al. Insular cortical and amygdaloid fibers are in contact with posterolateral hypothalamic neurons projecting to the nucleus of the solitary tract in the rat. Brain Res. 2006;1070: $139-144$.

26. Isnard J, Magnin M, Jung J, et al. Does the insula tell our brain that we are in pain? Pain. 2011;152:946.

27. Stehberg J, Levy D, Zangen A. Impairment of aversive memory reconsolidation by localized intracranial electrical stimulation. Eur J Neurosci. 2009;29:964-969.

28. Jensen J, McIntosh AR, Crawley AP, et al. Direct activation of the ventral striatum in anticipation of aversive stimuli. Neuron. 2003;40:1251.
Journal of Pain Research

\section{Publish your work in this journal}

The Journal of Pain Research is an international, peer-reviewed, open access, online journal that welcomes laboratory and clinical findings in the fields of pain research and the prevention and management of pain. Original research, reviews, symposium reports, hypothesis formation and commentaries are all considered for publication.

\section{Dovepress}

The manuscript management system is completely online and includes a very quick and fair peer-review system, which is all easy to use. Visit http://www.dovepress.com/testimonials.php to read real quotes from published authors. 